\title{
Spin and Orbital Moments of Ultra-Thin Fe Films on Various Semiconductor Surfaces
}

\author{
Jill S. Claydon ${ }^{1}$, Daxin $\mathrm{Niu}^{1}$, Yongbing $\mathrm{Xu}^{2}$, Neil D. Telling ${ }^{3}$, Ian W. Kirkman ${ }^{4}$, and Gerrit van der Laan ${ }^{3}$ \\ ${ }^{1}$ The Spintronics Laboratory, Department of Electronics, University of York, York YO10 5DD, U.K. \\ ${ }^{2}$ The Department of Electronics, University of York, York YO10 5DD, U.K. \\ ${ }^{3}$ Magnetic Spectroscopy Group of Daresbury Laboratory, Warrington WA4 4AD, U.K. \\ ${ }^{4}$ Station Scientist of Station 1.1 Daresbury Laboratory, Warrington WA4 4AD, U.K.
}

\begin{abstract}
The magnetic moments of ultrathin Fe films on three different III-V semiconductor substrates, namely $\mathrm{GaAs}_{\mathrm{An}} \mathrm{InAs}_{\mathrm{and}} \mathrm{In}_{0.2} \mathrm{Ga}_{0.8} \mathrm{As}$ have been measured with $X$-ray magnetic circular dichroism at room temperature to assess their relative merits as combinations suitable for next-generation spintronic devices. The results revealed rather similar spin moments and orbital moments for the three systems, suggesting the relationship between film and semiconductor lattice parameters to be less critical to magnetic moments than magnetic anisotropy.
\end{abstract}

Index Terms_Fe/InGaAs, interface magnetism, spin electronics, ultrathin films.

\section{INTRODUCTION}

$\mathbf{N}$ EXT-GENERATION spintronic devices are expected to revolutionize conventional electronic circuitry by utilizing both the charge and spin of the electron while retaining links with present day semiconductor technologies [1]-[3]. However, the development of such devices is at present limited by our abilities in transporting spin into nonmagnetic media [4], [5]. And so research into breaching the gap in characteristics between the magnetic materials and semiconductor technologies has become a hot topic of debate. Several technologies have been put forward to overcome this barrier such as magnetic semiconductors [5], where great advances have brought Curie temperatures up to $173 \mathrm{~K} \mathrm{[6]}$ and methods based on ferromagnet/semiconductor contacts [5], including epitaxially grown ferromagnetic metal contacts, which appear to offer the promise of room temperature spin transport.

After many years of investigation [7]-[11], it has recently been shown that ferromagnetic films have the capability to retain magnetic properties to interface thicknesses [12]. However, with predicted levels of spin transport still to be realized [4], many questions still remain unanswered about the degree to which interface magnetism and hence spin transport relates to the stability of growth and hence to the lattice match and surface of the materials involved.

In order to determine whether the property of lattice match and related film strain might be important for magnetic behavior close to the interface we have undertaken room temperature $\mathrm{X}$-ray magnetic circular dichroism (XMCD) investigations into the properties of ultrathin films grown on substrates of varied lattice constant. The substrates chosen for study, GaAs, InAs, and $\mathrm{In}_{0.2} \mathrm{Ga}_{0.8}$ As were chosen for their lattice constants which, in a $2: 1$ relationship with $\mathrm{Fe}$ give mismatches of $-1.56 \%$, $5.32 \%$, and $0 \%$, respectively.

\section{SAMPLE FABRication AND MEASUREMENT}

The specialist substrates used to establish the different lattice matches were constructed by the EPSRC's UK National Centre for III-V Semiconductor Technologies in Sheffield and took the form of GaAs (100) wafers topped with a $0.3 \mu \mathrm{m}$ epilayer of GaAs, InAs or InGaAs followed by an As capping layer. The As capping layer allowed for safe transit between systems and removed the requirement for pre-growth wet etching usually required to achieve the reconstructed surfaces.

Once in our own MBE system all substrates were initially heated to $350^{\circ} \mathrm{C}$ to desorb the As capping layer and then further annealed for $1 \mathrm{~h}$ to $560^{\circ} \mathrm{C}$ for $\mathrm{GaAs}, 525^{\circ} \mathrm{C}$ for InGaAs and $514^{\circ} \mathrm{C}$ for InAs RHEED analysis of the surface indicated an As rich $2 \times 4$ reconstruction was achieved for GaAs and InAs while a $1 \times 1$ surface was observed for $\operatorname{In}_{0.2} \mathrm{Ga}_{0.8}$ As. Following cooling to room temperature $7 \mathrm{ML} \mathrm{Fe}$, chosen for the large uniaxial anisotropy displayed at this thickness for the $\mathrm{Fe} / \mathrm{GaAs}$ system [15], was deposited at a rate of $0.66 \mathrm{ML}$ per minute, followed by the $15 \mathrm{ML}$ Cr cap to protect against oxidation. All stages of growth were monitored by RHEED measurement and the samples' uniaxial magnetic qualities were confirmed ex situ with longitudinal MOKE measurements.

XMCD measurements were conducted at station 1.1 of the Synchrotron Radiation Source at Daresbury Laboratory (U.K.) which was equipped with a flipper magnet with a maximum field of $0.6 \mathrm{~T}$. This configuration allows illumination of the samples with $85 \%$ circularly polarized X-rays parallel/antiparallel to an applied magnetic field and attainment of XAS spectra measured in these opposite applied field conditions, $I^{+}$and $I^{-}$. The flipper magnet offers the ability to reverse the magnetization at each measurement point and thus reduces anomalies in the XMCD or difference spectra. Measurements were made with the samples at an angle of $45^{\circ}$ to the incident $\mathrm{X}$-rays to maximize the effect of both the incident light and the applied field.

\section{RESUlTS AND DISCUSSION}

During growth RHEED patterns were obtained both prior and subsequent to the deposition of $\mathrm{Fe}$ on the reconstructed semi- 


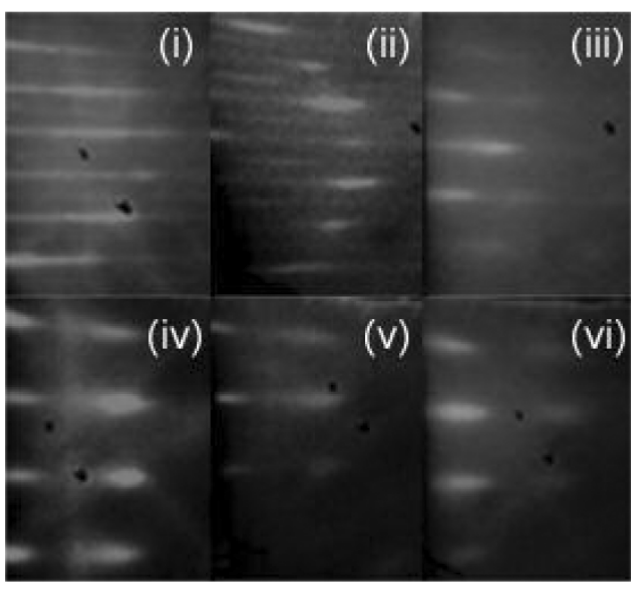

Fig. 1. RHEED images collected before (i, ii, iii) and after (iv, v, vi) deposition of $7 \mathrm{ML} \mathrm{Fe}$ onto the respective reconstructed $\mathrm{GaAs}$, InAs, and $\mathrm{In}_{0.2} \mathrm{Ga}_{0.8}$ As surfaces.

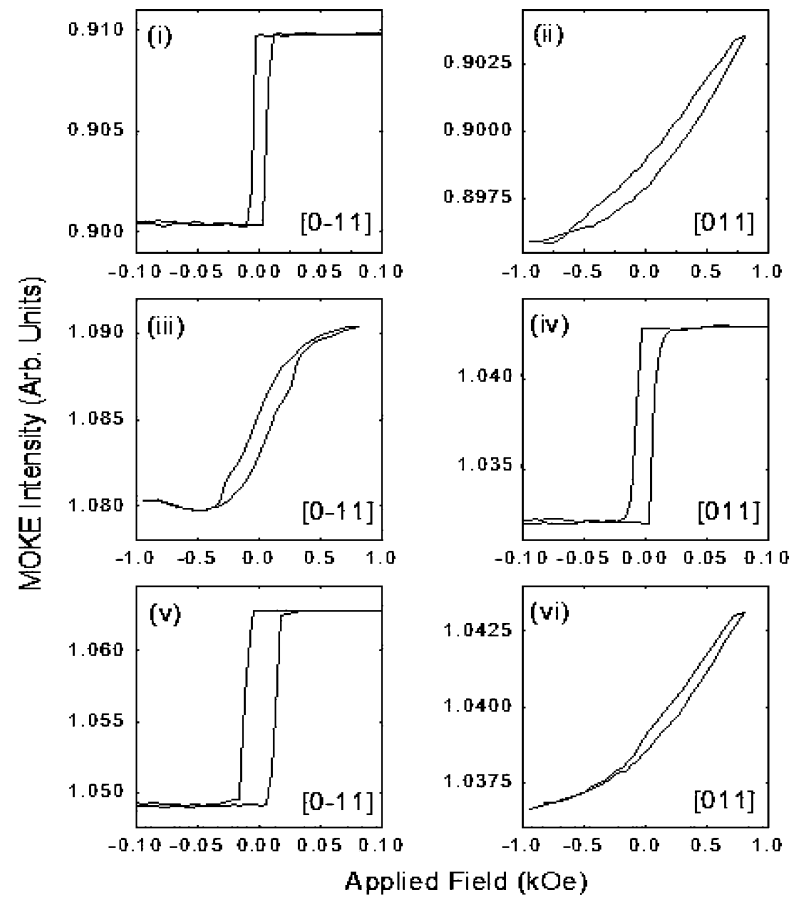

Fig. 2. MOKE loops taken along, the easy (i, iv, and v) and hard (ii, iii, and vi) axes of the $\mathrm{Fe} / \mathrm{GaAs}, \mathrm{Fe} / \mathrm{InAs}$, and $\mathrm{Fe} / \mathrm{In}_{0.2} \mathrm{Ga}_{0.8} \mathrm{As}$ samples respectively, show uniaxial anisotropy in all samples. Note the $90^{\circ}$ rotation of hard and easy axes in the case of $\mathrm{Fe} / \mathrm{InAs}$.

conductor surfaces. The images, which are shown in Fig. 1, confirm the epitaxial growth of bcc Fe on the three different surfaces with the same epitaxial relationship of $\mathrm{Fe}(100)<001>$ | $\mathrm{In}_{\mathrm{x}} \mathrm{Ga}_{1-\mathrm{x}} \mathrm{As}(100)\langle 001\rangle$, where $\mathrm{x}=0,1,0.2$.

Ex situ longitudinal MOKE measurements were also made, shown in Fig. 2. Although the hard axis of the Fe/GaAs and the $\mathrm{Fe} / \mathrm{In}_{0.2} \mathrm{Ga}_{0.8}$ As films could not be saturated, the hysteresis loops clearly show the films to be uniaxial in nature. An interesting aspect of these results is the confirmation of the reversal of easy axis and hard axis directions between the GaAs and InAs substrates. This result is in good agreement with previous studies [14] and is thought to be indicative of the influence of the magneto-elastic effect in the establishment of the uniaxial anisotropy. The magneto-elastic effect occurs as a direct

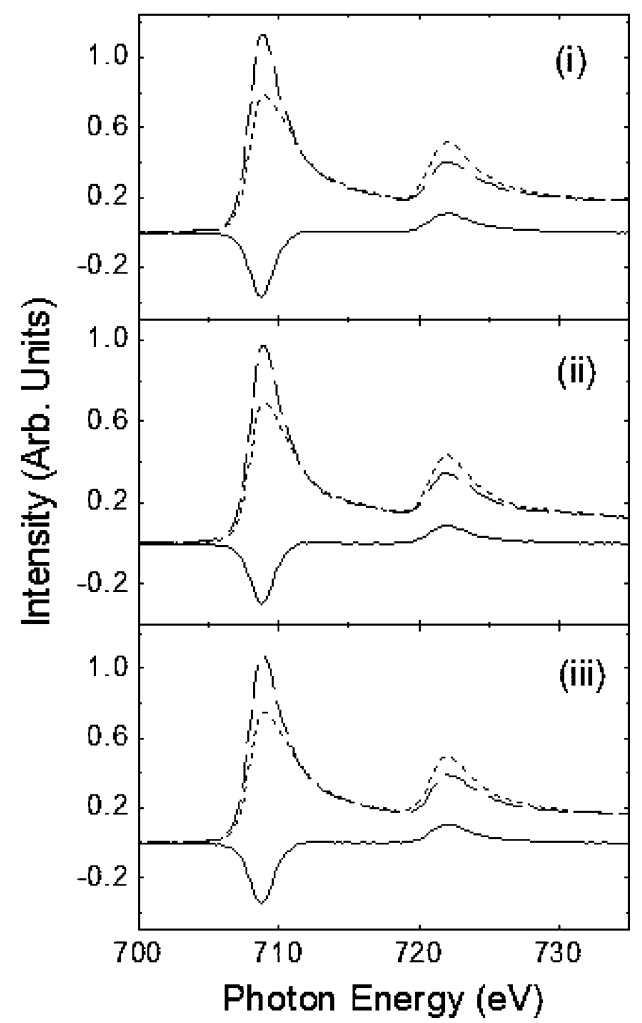

Fig. 3. XMCD spectra for $F e \mathrm{~L}_{2,3}$ edge studies of 7 ML Fe on GaAs (i), InAs (ii) and $\mathrm{In}_{0.2} \mathrm{Ga}_{0.8}$ As (iii). Solid lines correspond to XMCD spectra, while dashed and dotted lines represent the XAS spectra taken for opposite applied fields $I^{+}$and $I^{-}$spectra, respectively.

result of the compression or expansion of the Fe film in order to fit the GaAs or InAs lattice respectively. However, in these results we can also see that the perfectly lattice matched system $\mathrm{Fe} / \mathrm{In}_{0.2} \mathrm{Ga}_{0.8}$ As also displays uniaxial characteristics. While this can not be explained by magneto-elastically induced uniaxial anisotropy the result would seem to support the "chemical" effect proposed by Kneedler et al. [9] in which the uniaxial magnetic anisotropy (UMA) is derived from the unidirectional nature of Fe-As or Fe-Ga bonds. The results show that both chemical and magneto-elastic effects are in some part responsible for the observed UMA in Fe/III-V semiconductor heterostructures.

The obtained XMCD spectra, shown in Fig. 3, were analyzed through application of the sum rules, see (1)-(2), with the values of the parameters $p, q$, and $r$ derived from the integrals of the $\mathrm{XMCD}$ and XAS spectra in the manner of Chen et al. [15]. The number of holes, $\mathrm{n}_{h}$, was taken as 3.39 for $\mathrm{Fe}[16]$ and the degree of polarization, $P$, was equal to 0.85

$$
\begin{aligned}
m_{\text {orb }} & =-4 q n_{h} / 3 r P \\
m_{\text {spin }} & =(4 q-6 p) n_{h} / r P .
\end{aligned}
$$

The values of the spin and orbital moments found from sum rule analysis and those of the bulk values are described in Table I. By far the most striking aspect of the XMCD results is, within error, the similarity of observed magnetic moments between the three different substrates.

The highest spin moment of all the samples tested is seen for $\mathrm{Fe} / \mathrm{GaAs}$, however, this represents only $72 \%$ of the bulk value. This reduction compared with previous results on Ga rich 
TABLE I

SPIN AND ORBITAL MOMENTS OF BULK Fe IN COMPARISON TO 7 ML Fe FILM ON GaAs, InAs, AND $\operatorname{In}_{0.2} \mathrm{Ga}_{0.8}$ As

\begin{tabular}{cccc}
\hline \hline Sample & $m_{\text {orb }}\left(\mu_{\mathrm{B}}\right)$ & $m_{\text {spin }}\left(\mu_{\mathrm{B}}\right)$ & $m_{\text {orb }} / m_{\text {spin }}$ \\
\hline $\mathrm{Bulk \textrm {Fe } [ 6 ]}$ & 0.086 & 1.98 & 0.043 \\
$\mathrm{Fe} / \mathrm{GaAs}$ & $0.184 \pm 0.025$ & $1.43 \pm 0.18$ & $0.129 \pm 0.021$ \\
$\mathrm{Fe} / \mathrm{InAs}$ & $0.151 \pm 0.038$ & $1.35 \pm 0.17$ & $0.112 \pm 0.026$ \\
$\mathrm{Fe} / \mathrm{In}_{0.2} \mathrm{Gaa}_{0.8} \mathrm{As}$ & $0.186 \pm 0.021$ & $1.42 \pm 0.24$ & $0.131 \pm 0.026$ \\
\hline
\end{tabular}

$4 \times 6$ surface [17], [18] may originate from the different nature of the interface for these samples, which were grown on As rich reconstructions in this study, thought to give rise to reacted phases and hence reduced Fe moments [19]. It is also possible that the clustered growth phase that occurs in $\mathrm{Fe}$ at this thickness just prior to the establishment of a continuous film [12], [20] could require a higher external field, than was available to fully saturate these films. The $\mathrm{Fe} / \mathrm{GaAs}$ result is commensurate with the similarly reduced values of spin moment derived from $\mathrm{Fe} / \mathrm{InAs}$ and $\mathrm{Fe} / \mathrm{In}_{0.2} \mathrm{Ga}_{0.8} \mathrm{As}$, which are in close agreement with the $\mathrm{Fe} / \mathrm{GaAs}$ result representing $68 \%$ and also $72 \%$ of the bulk value, respectively.

In contrast to the reduction in spin moment the orbital moment, and hence the ratio $m_{\text {orb }} / m_{\text {spin }}$, for all samples shows a marked enhancement, with an orbital increase of around 200\%, with respect to the bulk value, as witnessed in earlier studies of $\mathrm{Fe} / \mathrm{GaAs}$ and $\mathrm{Fe} / \mathrm{InAs}$ [17], [18]. This orbital enhancement is thought to derive from the brake-down in symmetry close to the interface as a result of qualities of the substrate surface including steps or terraces and surface roughness. It is expected that these features cause localization of the $3 \mathrm{~d} F e$ wave functions close to the interface [21], our evidence would appear to support this since such surface issues would not be overcome by a perfect lattice match.

\section{CONCLUSION}

The spin and orbital moments and hysteresis loops of 7 ML Fe films grown on specially fabricated GaAs, InAs and $\mathrm{In}_{0.2} \mathrm{Ga}_{0.8}$ As epilayers have been measured using XMCD and MOKE, respectively. The results show that magneto-elastic effects provoked by the difference in substrate lattice constant have little effect on the spin and orbital moments which are found to be within error of one another for all substrates. The tolerance of the Fe films to withstand such magneto-elastic effects might further suggest $\mathrm{Fe} / \mathrm{III}-\mathrm{V}$ semiconductor heterostructures could, in future provide suitable solutions for spin-transport devices and hints that the flexibility of these systems may offer practical manufacturing solutions in the field of future spintronics device design.

\section{ACKNOWLEDGMENT}

This work was supported in part by an EPSRC Advanced Fellowship and a CASE studentship awarded by Daresbury Laboratory.

\section{REFERENCES}

[1] G. A. Prinz, "Hybrid ferromagnetic-semiconductor structures," Science, vol. 250, no. 23, pp. 1092-1097, 1990.

[2] S. Datta and B. Das, "Electronic analog of the electro-optic modulator," Appl. Phys. Lett., vol. 56, no. 7, pp. 665-667, 1990.

[3] S. A. Wolf, D. D. Awschalom, R. A. Buhrman, J. M. Daughton, S. von Molnar, M. L. Roukes, A. Y. Chtchelkanova, and D. M. Treger, "Spintronics: A spin based electronics vision for the future," Science, vol. 294, pp. 1488-1495, Nov. 2001.

[4] B. T. Jonker, "Progress toward electrical injection of spin-polarized electrons into semiconductors," Proc. IEEE, vol. 91, no. 5, pp. 727-740, May 2003.

[5] S. A. Chambers and Y. K. Koo, "New materials for spintronics," MRS Bull., vol. 28, p. 706, 2003.

[6] K. Wang et al., "Magnetism in (Ga, Mn)As thin films with $\mathrm{T}_{C}$ up to 173 K," ICPS-27 Conf. Proc., to be published.

[7] J. J. Krebs, B. T. Jonker, and G. A. Prinz, "Properties of Fe single-crystal films grown on (100)GaAs by molecular-beam epitaxy," J. Appl. Phys., vol. 61, no. 7, pp. 2596-2599, 1987.

[8] A. Filipe, A. Schuhl, and P. Galtier, "Structure and magnetism of the Fe/GaAs interface," Appl. Phys. Lett., vol. 70, no. 1, pp. 129-131, 1997.

[9] E. M. Kneedler, B. T. Jonker, P. M. Thibado, R. J. Wagner, B. V. Shanabrook, and L. J. Whitman, "Influence of substrate surface reconstruction on the growth and magnetic properties of $\mathrm{Fe}$ on $\mathrm{GaAs}(001)$," Phys. Rev. B, vol. 56, no. 13, pp. 8163-8168, 1997.

[10] Y. B. Xu, E. T. M. Kernohan, D. J. Freeland, A. Ercole, M. Tselepi, and J. A. C. Bland, "Evolution of the ferromagnetic phase of ultrathin Fe films grown on GaAs(100)-4 × 6," Phys. Rev. B, vol. 58, no. 2, pp. 890-895, 1998.

[11] F. Bensch, G. Garreau, R. Moosbühler, G. Bayreuther, and E. Beaurepaire, "Onset of ferromagnetism in Fe epitaxially grown on $\mathrm{GaAs}(001)$ $(4 \times 2)$ and $(2 \times 6)$, , J. Appl. Phys., vol. 89, no. 11 , pp. 7133-7135, 2001.

[12] J. S. Claydon, Y. B. Xu, M. Tselepi, J. A. C. Bland, and G. van der Laan, "Direct observation of a bulklike spin moment at the Fe/GaAs(100)-4 × 6 interface," Phys. Rev. Lett., vol. 93, no. 3, p. 37206, 2004.

[13] M. Brockmann, M. Zölfl, S. Miethaner, and G. Bayreuther, "In-plane volume and interface magnetic anisotropies in epitaxial $\mathrm{Fe}$ films on GaAs(001)," J. Magn. Magn. Mater, vol. 199, pp. 384-386, Jun. 1999.

[14] Y. B. Xu, D. J. Freeland, M. Tselepi, and J. A. C. Bland, "Uniaxial anisotropy of epitaxial Fe films on $\operatorname{InAs}(100)-4 \times 2$ and GaAs(100)-4 × 2," J. Appl. Phys., vol. 87, pp. 6110-6113, May 2000.

[15] C. T. Chen, Y. U. Idzerda, H.-J. Lin, N. V. Smith, G. Meigs, E. Chaban, G. H. Ho, E. Pellegrin, and F. Sette, "Experimental confirmation of the $\mathrm{X}$-ray magnetic circular dichroism sum rules for iron and cobalt," Phys. Rev. Lett., vol. 75, no. 1, pp. 152-155, 1995.

[16] P. Carra, B. T. Thole, M. Altarelli, and X. Wang, "X-ray circular dichroism and local magnetic fields," Phys. Rev. Lett., vol. 70, no. 5, pp. 694-697, 1993.

[17] Y. B. Xu, M. Tselepi, C. M. Guertler, C. A. F. Vaz, G. Wastblauer, J. A. C. Bland, E. Dudzik, and G. van der Laan, "Giant enhancement of orbital moment and perpendicular anisotropy in epitaxial Fe/GaAs(100)," J. Appl. Phys., vol. 89, no. 11, pp. 7156-7158, 2001.

[18] Y. B. Xu, M. Tselepi, J. Wu, S. Wang, J. A. C. Bland, Y. Huttel, and G. van der Laan, "Interface magnetic properties of epitaxial Fe-InAs heterostructures," IEEE Trans. Magn., vol. 38, no. 5, pp. 2652-2654, Sep. 2002.

[19] S. Mirbt, B. Sanyal, C. Isheden, and B. Johansson, "First-principles calculations of Fe on GaAs(100)," Phys. Rev. B, vol. 67, p. 155421, 2003.

[20] I. M. L. Billas, A. Châtelain, and W. A. de Heer, "Magnetism from the atom to the bulk in iron, cobalt and nickel clusters," Science, vol. 265 , pp. 1682-1684, 1994.

[21] G. van der Laan, "Magnetic linear X-ray dichroism as a probe of the magnetocrystalline anisotropy," Phys. Rev. Lett., vol. 82, pp. 640-643, 1999.

Manuscript received February 6, 2005. 